\title{
Caring for Confused Community Dwelling Seniors: Contributing and Obstructing Factors in Daily Care for Healthcare Assistants in District Nursing
}

\author{
Bosma $\mathrm{C}^{1 *}$, Ketelaar $\mathrm{N}^{2}$ and Rouwette-Witting $\mathbf{M}^{3}$ \\ ${ }^{1}$ Organization for elderly care, Carintreggeland, Netherlands \\ ${ }^{2}$ Research group Social Work, Saxion University of Applied Sciences, Netherlands \\ ${ }^{3}$ Research group Social Work, Saxion University of Applied Sciences, Netherlands \\ *Correspondence to: Corina Bosma, Organization for Health and Social Care, Carintreggeland, Netherlands; E-mail: corina.bosma@carintreggeland.nl
}

Received: February 25, 2019; Accepted: March 10, 2019; Published: April 20, 2019;

\begin{abstract}
Older people with confused behavior, have behavioral problems due to dementia, mental problems or social problems. For the Healthcare Assistant (HA) in district nursing, it is a daily challenge to care for older people with confused behavior. Aim of this research is to achieve an insight in the strategies the HA uses to deal with the daily care for older people with confused behavior. It is also the aim to have insight in factors which contribute to the daily care in a positive or negative way. Method: a qualitative explorative research. 17 HA's in district nursing participated in semi-structured interviews. All respondents had experience with caring for older people with confused behavior. The most important influencing factors are the experienced relationship between HA and the client and the experienced support by the team. Particularly behavioral problems due to mental problems can impede a relationship with the client. Further research is recommended to study the level of knowledge and competences of all levels of employees in district nursing.
\end{abstract}

Keywords: Confused persons, vulnerable elderly, older adults living at home, mental disorder, home care staff, district nursing

\section{Short Commentary}

The number of seniors confronted by chronic illness and dementia is rising, due to the fact that people are getting older and stay at home longer. Because of rising healthcare costs, people are to assumed to stay at home as long as possible [1]. Though, age-related physical and cognitive decline can make aging at home challenging [2]. Psychiatric problems among community dwelling seniors is frequently recognized by healthcare assistants (HA's) in district nursing [2]. One of the consequences of these problems is an increase of the socalled confused community dwelling seniors. Seniors with confused behaviour show problems like agitation, aggression or apathy. For HA's it is a daily challenge to manage the care for confused community dwelling seniors. It is important that HA's have sufficient knowledge of confused behaviour in seniors in order to recognize and manage psychiatric problems [2]. However, little is known about what HA's experience themselves as contributing or obstructing factors to daily care and how to respond in a right way to confused behaviour as well as to provide good care. We conducted an explorative research among seventeen HA's in the Netherlands to gain insight in these contributing and obstructing factors. The qualified HA's, with a variety in age and work experience, provide basic personal care under supervision of a district nurse.

The professionals were asked, in an individual interview, to share their experiences with confused community dwelling seniors and how they manage daily care. The Cohen-Mansfield mapping of problem behaviour, an instrument for assessing agitation, was used to stimulate participants to give more detailed information and examples of problem behaviour they experienced [3]. Most of the participants experience an increase of confused community dwelling seniors, which was in line with research conducted by Grundberg and colleagues concerning the role of home care assistants recognizing mental health problems in community dwelling seniors [4]. HA's reported daily difficulties and challenges in caring for confused community dwelling seniors. The HA's were confronted with verbal aggressive behaviour like threats, berate or curse by the seniors. They were also confronted with physically non-aggressive behaviour like compulsive or risky behaviours of the seniors. According to the HA's, there is no 'standard recipe' to manage problem behaviour: the way the HA's approach confused seniors depends on factors related to the seniors as well as characteristics of the HA. These factors are for example age and work experience of the professional as well as the type and cause of behaviour problem of the senior. The presence or absence of a relation of trust, information and support are the main contributing or obstructing factors.

Despite the fact that a relation of trust is seen as essential for providing good care, it seems very difficult to achieve such a relation with confused community dwelling seniors. According to the HA's, a relation of trust can be reached when they have the opportunity to get to know the client. HA's experience more difficulties to become 
familiar with the senior and building up a relation of trust in case a diagnose is missing and psychiatric problems are suspected. A study of Gleason and Coyle regarding the experiences of home care workers providing homecare to clients diagnosed with mental and behavioural problems, also mentioned the struggles in building a relation of trust in such cases [5]. In addition, in cases where psychiatric problems are suspected, the HA's in our study seemed to have less tendency to build this relation of trust. Several factors might explain this low tendency: having a primary focus on personal care and not on a relation of trust, uncertainty of the HA or not having the required knowledge on how to respond to clients with (suspected) psychiatric diagnoses. These factors are also identified in a study on how nursing staff in a hospital manage patients with psychiatric problems [6]. Also a lack of collaboration with professionals in social care can obstruct a relation of trust [7].

The second factor, as mentioned by the HA's, is the presence or absence of information on the underlying cause of the behaviour problem, for example dementia or loneliness of the senior. Olivera and colleagues studied the factors contributing to the development of psychiatric problems in community dwelling seniors [8]. When professionals in home care are aware of these factors, problems could be recognized at an early stage. In our study, HA's stated that when information about the cause and reason of the behaviour is available, this increases their ability to recognize the problems. In such cases, HA's experience a better management of problem behaviour and they feel like they succeed (more) in providing daily care.

At last, the HA's perceive the available support of the district nursing team as a contributing or obstructing factor in the daily care for confused community dwelling seniors. Important is sharing knowledge and experiences within the team and with other professionals in healthcare and social care The importance of exchanging knowledge and experiences is confirmed in a study by Gleason and Coyle [5]. When confronted with problem behaviour, (emotional) support of other experts seems very important. Of course, attention is also still needed by strengthen (theoretical) knowledge about psychiatric problems and how to recognize such problems.

Stay longer at home by seniors, including confused community dwelling seniors might be desired and needed because of rising health costs. Though professionals both health and social care need help and more attention to overcome contributing and obstructing factors. Specifically providing information and (emotional) support, can support professionals in district nursing and can increase their feeling of being capable of building up a relation of trust with confused community dwelling seniors. Subsequently, this relation of trust can contribute to the provision of optimal care to the client. Development of further collaboration of health care and social care professionals can contribute to more optimal and integrated care for both personal and mental health care $[1,7]$.

Note: Accepted for publication in the Dutch Journal of Gerontology and Geriatrics in Dutch (Tijdschrift voor Gerontologie en Geriatrie).

\section{References}

1. Josefsson K, Meranius, MS (2018) Complexity in daily living of older adults with multimorbidity: health, social and informal care utilization and costs. J Gerontol Geriatr Med 4:017.
2. Haddad M, Plummer S, Taverner A, Gray R, Lee S, Payne F, Knight D (2005) District nurses' involvement and attitudes to mental health problems: a three-area cross-sectional study. J Clin Nursing 14: 976-985. [Crossref]

3. Cohen-Mansfield J, Marx MS, Rosenthal AS (1989) A description of agitation in a nursing home. J Gerontology 44: 77-84. [Crossref]

4. Grundberg A, Hansson A, Religa D, Hilleras P (2016) Home care assistants perspectives on detecting mental health problems and promoting mental health among community-dwelling seniors with multimorbidity. J Multidisciplinary Healthcare 9: 83-95. [Crossref]

5. Gleason HP, Coyle CE (2016) Mental and behavioral conditions among older adults: implications for the home care workforce. Aging Mental Health 20: 848855. [Crossref]

6. Lethoba KG, Netswera FG, Rankhumise E (2006) How professional nurses in a general hospital setting perceive mental ill patients. Curationis, 29: 4-11. [Crossref]

7. Rämgård M, Blomqvist K, Petersson P (2015) Developing health and social care planning in collaboration. J Interprofessional Care, 29: 354-358. [Crossref]

8. Olivera J, Benabarre S, Lorente T, Rodrigues M, Barros A, Quintana C, Pelegrina V, Aldea C (2011) Detecting psychogeriatric problems in primary care: factors related to psychiatric symptoms in older community patients. $J$ Mental Health in Family Medicine 8: 11-19. [Crossref]

\section{Citation:}

Bosma C, Ketelaar N and Rouwette-Witting M (2019) Caring for Confused Community Dwelling Seniors: Contributing and Obstructing Factors in Daily Care for Healthcare Assistants in District Nursing. Ageing Sci Ment Health Stud Volume 3(2): 1-2. 